\title{
Improving regional dose distribution while maintaining high delivery efficiency for hypofractionated whole breast radiotherapy
}

\author{
Lijun $\mathrm{Ma}^{1}$, Andrew Hwang ${ }^{2}$ \\ ${ }^{1}$ Department of Radiation Oncology, University of California San Francisco, San Francisco, CA, USA; ${ }^{2}$ Department of Radiation Oncology, Palo \\ Alto Medical Foundation, Palo Alto, CA, USA \\ Correspondence to: Lijun Ma, PhD, FAAPM. UCSF Radiation Oncology, 505 Parnassus Ave, Room L08, San Francisco, CA 94143, USA. \\ Email: Lijun.Ma@ucsf.edu.
}

\begin{abstract}
Skin toxicities, particularly in the inframammary fold areas are noted for patients receiving hypofractionated whole breast radiation therapy. In this study, a hybrid modulated beam technique was developed to tackle such a problem. The technique aimed to achieve significant dose improvements to the inframammary region while maintaining the robustness and treatment delivery efficiency similar to that of $3 \mathrm{D}$ conformal treatment delivery. The overall treatment time for the technique was about 6 minutes or less for a treatment session delivering a fractional dose of $266 \mathrm{cGy}$. As a result, clinical centers with a high volume of patients are most likely to benefit from implementing such a technique.
\end{abstract}

Keywords: Whole breast irradiation; treatment planning optimization; inframammary fold

Received: 26 July 2018; Accepted: 07 November 2018; Published: 12 December 2018.

doi: $10.21037 /$ tro.2018.11.01

View this article at: http://dx.doi.org/10.21037/tro.2018.11.01

\section{Introduction}

The general goal of whole breast radiation therapy is to deliver a uniform dose to the irradiated area and avoid treatment-related toxicities (1-9). Traditionally, this is done by using opposed tangential fields with wedges to improve dose homogeneity. Several techniques have been reported on optimizing individual beam segments based on tangential beams to achieve such a goal $(8,10-12)$. Pros and cons of these techniques have been studied $(5,8,13-15)$. The major advantage of these techniques is the improved dosimetry and the major concerns include increased planning effort and workflow efficiency. For routine clinical practice, a recent study has advocated the use of $3 \mathrm{D}$ conformal techniques over intensity-modulated beams as the firstline treatment of the whole breast due to equivalence in observed acute toxicities (16). As a result, some high-volume clinical centers in the world continue to adopt the workflow of 3D conformal treatments for the whole breast treatment.

When dealing with acute skin toxicities for consideration of adopting hypofractionated treatments involving shortened treatment course and increased fractional dose (17-21), large breast size has been reported to contribute to increased skin toxicities $(22,23)$. For such patients, 3D conformal treatment has become challenging to avoid focal skin toxicities in critical areas. In particular, significant isodose hot spots tend to occur in multiple areas toward the inferior portion of the breast. This has raised the concern for focal skin toxicities near the inframammary sulcus where quality of life of the patients can be severely affected due to sensitivity of the area.

With the goal of consistently treating all patients without sacrificing delivery efficiency in a remote high-volume clinical setting, we specifically developed a mixed beam approach by combining small intensity modulated beam segments with large open tangential fields for possible hypofractionated whole-breast treatments. The small beam segments aim to provide dose hot spot compensation while the large open fields aim to provide necessary baseline dose and skin-flashing that facilitates online portal verifications for a rapid treatment setup similar to that of $3 \mathrm{D}$ conformal treatment.

With an open field contribution, we hypothesize that the total number of segments for the modulated fields would remain adequate in order to match the treatment delivery efficiency of $3 \mathrm{D}$ conformal treatments despite 
higher fractional dose required for hypofractionated treatments. Maintaining a high delivery efficiency not only improves patient throughputs but also minimizes treatment setup uncertainties. The goal of our study is therefore to investigate whether such a mixed beam technique would achieve high efficient delivery while improving the dose uniformity especially to the inframammary fold area for large breasted patients.

\section{Methods and materials}

Six patient cases with the planning target volume ranging from 1,350 to $4,202 \mathrm{cc}$ and separation $>25 \mathrm{~cm}$ were selected and tested for the technique. All cases were anonymized prior to the selection and they were retrospectively replanned for a fractional dose of $266 \mathrm{cGy}$ intended for a course of 15 or 16 fractions of treatment. As standard practice, all patients underwent thin-cut CT scan with the breast board and an aquaplast chest mask immobilizing the whole breast. Following the standard clinical practice, the initial tangential fields were placed via virtual simulation using a commercial treatment planning system (Pinnacle version 9, Philips Medical Solutions, OH). The tangential fields were placed to align with the medial and lateral markers placed on the chest to mark the field edges. For all cases, a 2-cm skin-flashing margin was added beyond the skin surface. The isocenter of all the fields was placed inside the breast and 1-2 cm from the lung interface and halfway between the superior and inferior border. The whole breast volume was contoured via the auto-contouring tool of the treatment planning system. In order to constrain the dose to the inframammary region for the current study, a partial constraining volume starting from the inferior border extending approximately $4 \mathrm{~cm}$ superiorly was contoured and the dose volume constraints were imposed on the area.

An automated script was programmed to streamline the planning process: the script first sets equal beam weights for the tangential fields. Then the maximum dose from the open-field was calculated. The monitor units for each beam were then scaled down by a constant factor (e.g., 0.6-0.8). As a starting value, the scaling factor was set to the inverse of maximum percentage dose of the open field contribution. For example, if the maximum percentage dose for the open field is $130 \%$, then the scaling factor is set to 0.77 (i.e., $1 / 130 \%$ ) for subsequent planning optimizations.

After scaling down the monitor units, two additional tangential fields of the same setup were added, and beam optimization was performed on these two fields based on the dose volume constraints of the whole breast, the partial inframammary fold region, the lung and the heart (left-sided treatment only). During the inverse planning optimization, the dose distribution from the open field is locked for the two open tangential fields, i.e., the intensity modulated beam optimization was performed over the background dose of the open tangential fields. Afterward, the contribution from the open field was iteratively adjusted and the above steps were repeated to achieve an optimal treatment plan.

The following dose volume constraints were employed for the inverse planning process: (I) the maximum percentage volume of the inframammary fold region receiving $>100 \%$ of the prescription dose is less than $2 \%$; (II) maximize the dose uniformity to the whole breast volume; (III) the maximum percentage of the whole breast volumes receiving $>105 \%$ of the prescription dose is less than $2 \%$; (IV) the minimum percentage volume of the whole breast volume receiving $>95 \%$ of the prescription dose is $>98 \%$.

In addition, the dose volume constraints for the lung and to the heart (for left-sided breast case) are given as follows: (I) the maximum percentage volume of the ipsilateral lung receiving $>75 \%$ of the prescription is less than $5 \%$; (II) the maximum percentage volume of the heart receiving $>75 \%$ of the prescription dose is less than $2 \%$.

In general, the entire planning process took about 1 minute to complete with the optimization yielding a total number of beam segments on the order of 10-30 for a given case. For each patient case, treatment plans were then benchmarked against the conventional wedge pair (WP) technique for a high-volume clinical setting, where the treatment time was limited to 10 minutes or less per treatment session.

\section{Results}

Figure 1 compares the dose distributions of the combined open and modulated beams versus the standard WP technique. From the results of Figure 1, the dose uniformity was significantly improved especially near the inferior and the superior part of the breast. The dose hot spots at the inframammary sulcus were significantly reduced: the $115 \%$ isodose line was not visible in Figure $1 B$ where a significant portion of the breast volume was enclosed as shown in Figure $1 A$. The dose conformity, i.e., the separation between the isodose lines, as well as the lung dose was also improved for the mixed-beam approach.

The dose volume histogram for the case is given in Figure 2. From the results of Figure 2, the overall plan quality is improved for the mixed beam approach versus the 

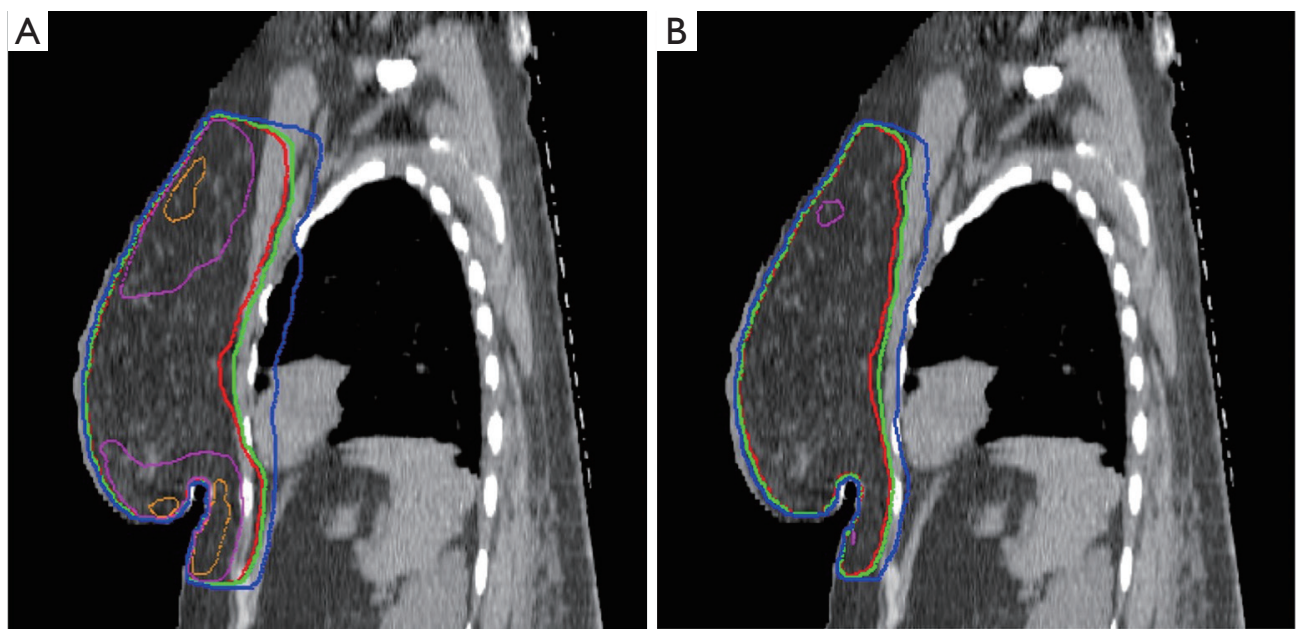

Figure 1 Isodose comparison of the standard wedge pair versus the intensity modulated mixed beam approach: (A) is the standard wedge pair technique and (B) is the mixed open-field and intensity modulated beam technique. The isodose lines shown are $115 \%, 110 \%, 100 \%$, $90 \%$ and $60 \%$ respectively.

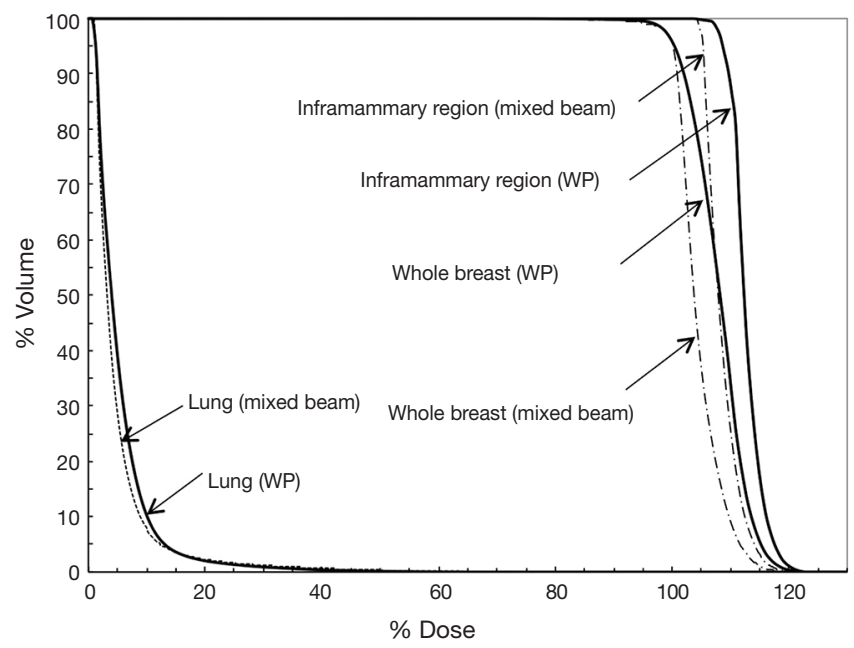

Figure 2 Dose volume histogram for the mixed beam approach compared with the standard wedge pair technique. Note significant differences for the whole breast and the lung.

WP technique. For example, the dose hot spots such as the whole breast volume enclosed by the $105 \%$ isodose line were reduced by $58.9 \%$. The dose coverage to the whole breast is equivalent between the two approaches. However, the dose near the inframammary sulcus region was all less than $105 \%$ for the mixed-beam approach. The total number of beam segments was 15 and the entire delivery was estimated to take about 6 minutes to complete for the mixed beam approach with $1.1 \mathrm{~min}$ beam-on time for a machine output of $600 \mathrm{MU} /$ min and an estimated treatment setup time of 5 minutes.

The results of all the studied cases are summarized in Table 1. From the results shown in Table 1, the mixed-beam approach significantly improves the dose uniformity for all the cases compared to the WP technique: the mean dose hot spot volume enclosed by the $105 \%$ prescription isodose line decreased from $54 \%$ to $24 \%$; the maximum dose to the inframammary sulcus area also decreased significantly from $116 \%$ to $104 \%$ based on the $\mathrm{P}$ value of 0.006 from the two-tailed paired student $t$-test. Small decrease $(\sim 0.5-4 \%)$ in the $\mathrm{D}_{20}$ to the lung $(\mathrm{P}=0.03)$ was also noted. However, the $\mathrm{P}$ values were not statistically significant for the mean lung dose $(\mathrm{P}=0.09)$ and $\mathrm{D}_{50}$ heart dose $(\mathrm{P}=0.053)$.

The contributions of the open field and the effect of scaling factor on the dose uniformity are illustrated in Figure 3. As shown in Figure 3, non-optimal value of the scaling factor degraded the dose uniformity to the whole breast volume. We noticed that when the contribution from the open field was set higher than the optimal value of $70 \%$ (such as $80 \%$ ), the background dose limited the amount of beam modulation possible. However, when the contribution from the open field was set lower than the optimal value (such as $50 \%$ ), modulated beams tend to compensate it by abutting multiple small beam segments thus resulting in a less uniform dose to the whole breast volume.

\section{Discussion}

A simple and efficient technique compatible with $3 \mathrm{D}$ 
Table 1 Summary of the patient cases treated with intensity mixed beam (IMB) technique as compared with standard wedge-pair (WP) technique: $V_{105 \%}$ denotes target volume receiving at least $105 \%$ of the prescription dose, lung $D_{\text {mean }}$ and $D_{20}$ denote the mean lung dose and the dose to at least $20 \%$ of the lung expressed in percentage of the prescription dose, heart $\mathrm{D}_{50}$ denotes the dose to at least $50 \%$ of the heart volume, IMF $\mathrm{D}_{\max }$ denotes the maximum dose at the inframammary fold (IMF) region

\begin{tabular}{|c|c|c|c|c|c|c|c|c|c|}
\hline Case No. & $\begin{array}{l}\text { Volume } \\
\text { (cc) }\end{array}$ & \multicolumn{2}{|c|}{$\mathrm{V}_{105 \%}(\%)$} & \multicolumn{2}{|c|}{ Lung $D_{\text {mean }} / D_{20}(\%)$} & \multicolumn{2}{|c|}{ Heart $D_{50}(\%)$} & \multicolumn{2}{|c|}{ IMF D $D_{\max }(\%)$} \\
\hline 1 (left) & 2,705 & 38 & 32.2 & $5.1 / 14.8$ & $5.2 / 14.2$ & 21.9 & 20.7 & 115 & 104 \\
\hline 2 (right) & 2,447 & 76 & 31.2 & $3.3 / 8.5$ & $2.9 / 6.1$ & 1.2 & 1.1 & 118 & 105 \\
\hline 3 (right) & 1,350 & 21.2 & 4.5 & $2.3 / 4.8$ & $2.0 / 4.4$ & 3.6 & 3.5 & 108 & 101 \\
\hline 5 (left) & 3,388 & 78.9 & 31.5 & $4.5 / 13.9$ & $3.5 / 9.7$ & 26.7 & 24.3 & 119 & 104 \\
\hline 6 (left) & 4,202 & 81 & 38.9 & $5.2 / 12.7$ & $5.1 / 10.3$ & 10.5 & 9.7 & 121 & 106 \\
\hline
\end{tabular}

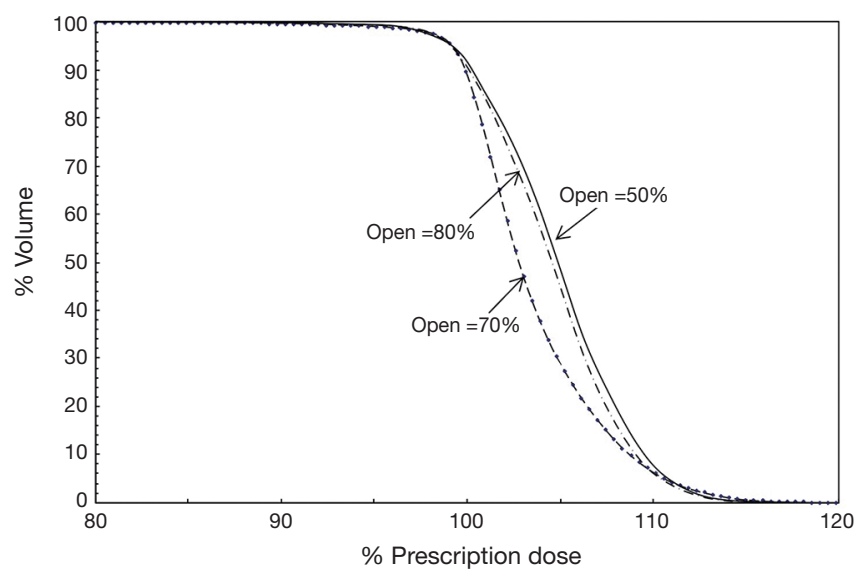

Figure 3 The effect of the scaling factor values on the target dose uniformity for the mixed open field and the intensity modulated field approach.

conformal treatment workflow has been developed for hypofractionated whole breast radiation therapy. For proof of concept, highly uniform dose $(<5 \%)$ toward the inframammary area was realized with the technique for six complex breast treatment cases.

The major strength of the technique is its targeted area dose sparing capability in conjunction with its high delivery efficiency that rivals $3 \mathrm{D}$ conformal WP treatment. This result is also supported by studies of similar techniques for conventional whole breast treatment $(24,25)$. One major distinction of our technique is that it explicitly varied the open field contribution to optimize the intensity modulated fields leading to approximately $30 \%$ to $50 \%$ of its contribution to eliminate dose hot spots specific to the inframammary fold area. Another strength of this technique is the simplicity of the planning process. The use of a standard script minimizes the additional planning time required.

With skin-flashing open field embedded in the technique, this technique adopts the same treatment setup and workflow as standard 3D conformal treatments. We expect that this technique would benefit a high-volume clinical center for implementing hypofractionated whole-breast radiotherapy treatments where standard $3 \mathrm{D}$ conformal workflow is mandatory for the practice.

\section{Acknowledgments}

Funding: None.

\section{Footnote}

Conflicts of Interest: Both authors have completed the ICMJE uniform disclosure form (available at http:// dx.doi.org/10.21037/tro.2018.11.01). The authors have no conflicts of interest to declare.

Ethical Statement: The authors are accountable for all aspects of the work in ensuring that questions related to the accuracy or integrity of any part of the work are appropriately investigated and resolved. All procedures performed in studies involving human participants were in accordance with the ethical standards of the institutional and/or national research committee(s) and with the Helsinki Declaration (as revised in 2013). No informed consent was obtained from the patients as the data was based on anonymized retrospective records. De-identified data were 
obtained for the study.

Open Access Statement: This is an Open Access article distributed in accordance with the Creative Commons Attribution-NonCommercial-NoDerivs 4.0 International License (CC BY-NC-ND 4.0), which permits the noncommercial replication and distribution of the article with the strict proviso that no changes or edits are made and the original work is properly cited (including links to both the formal publication through the relevant DOI and the license). See: https://creativecommons.org/licenses/by-nc-nd/4.0/.

\section{References}

1. Chui CS, Hong L, Hunt M, et al. A simplified intensity modulated radiation therapy technique for the breast. Med Phys 2002;29:522-9.

2. Demirci S, Nam J, Hubbs JL, et al. Radiation-induced cardiac toxicity after therapy for breast cancer: interaction between treatment era and follow-up duration. Int $\mathrm{J}$ Radiat Oncol Biol Phys 2009;73:980-7.

3. Freedman GM, Anderson PR, Li J, et al. Intensity modulated radiation therapy (IMRT) decreases acute skin toxicity for women receiving radiation for breast cancer. Am J Clin Oncol 2006;29:66-70.

4. Goodman KA, Hong L, Wagman R, et al. Dosimetric analysis of a simplified intensity modulation technique for prone breast radiotherapy. Int J Radiat Oncol Biol Phys 2004;60:95-102.

5. Haciislamoglu E, Colak F, Canyilmaz E, et al. The choice of multi-beam IMRT for whole breast radiotherapy in early-stage right breast cancer. Springerplus 2016;5:688.

6. Hong L, Hunt M, Chui C, et al. Intensity-modulated tangential beam irradiation of the intact breast. Int J Radiat Oncol Biol Phys 1999;44:1155-64.

7. Hurkmans CW, Cho BC, Damen E, et al. Reduction of cardiac and lung complication probabilities after breast irradiation using conformal radiotherapy with or without intensity modulation. Radiother Oncol 2002;62:163-71.

8. Vicini FA, Sharpe M, Kestin L, et al. Optimizing breast cancer treatment efficacy with intensity-modulated radiotherapy. Int J Radiat Oncol Biol Phys 2002;54:1336-44.

9. Kestin LL, Sharpe MB, Frazier RC, et al. Intensity modulation to improve dose uniformity with tangential breast radiotherapy: initial clinical experience. Int J Radiat Oncol Biol Phys 2000;48:1559-68.

10. Fraass BA, Kessler ML, McShan DL, et al. Optimization and clinical use of multisegment intensity-modulated radiation therapy for high-dose conformal therapy. Semin Radiat Oncol 1999;9:60-77.

11. Lo YC, Yasuda G, Fitzgerald TJ, et al. Intensity modulation for breast treatment using static multi-leaf collimators. Int J Radiat Oncol Biol Phys 2000;46:187-94.

12. Thilmann C, Zabel A, Nill S, et al. Intensity-modulated radiotherapy of the female breast. Med Dosim 2002;27:79-90.

13. Donovan EM, Yarnold JR, Adams EJ, et al. An investigation into methods of IMRT planning applied to breast radiotherapy. Br J Radiol 2008;81:311-22.

14. Schubert LK, Gondi V, Sengbusch E, et al. Dosimetric comparison of left-sided whole breast irradiation with 3DCRT, forward-planned IMRT, inverse-planned IMRT, helical tomotherapy, and topotherapy. Radiother Oncol 2011;100:241-6.

15. Zhang F, Zheng $M$. Dosimetric evaluation of conventional radiotherapy, 3-D conformal radiotherapy and direct machine parameter optimisation intensity-modulated radiotherapy for breast cancer after conservative surgery. J Med Imaging Radiat Oncol 2011;55:595-602.

16. Guttmann DM, Gabriel P, Kennedy C, et al. Comparison of acute toxicities between contemporary forward-planned $3 \mathrm{D}$ conformal radiotherapy and inverse-planned intensitymodulated radiotherapy for whole breast radiation. Breast J 2018;24:128-32.

17. Caudrelier JM, Truong PT. Role of hypofractionated radiotherapy in breast locoregional radiation. Cancer Radiother 2015;19:241-7.

18. Ciammella P, Podgornii A, Galeandro M, et al. Toxicity and cosmetic outcome of hypofractionated whole-breast radiotherapy: predictive clinical and dosimetric factors. Radiat Oncol 2014;9:97.

19. Ishihara T, Yoden E, Konishi K, et al. Long-term outcome of hypofractionated radiotherapy to the whole breast of Japanese women after breast-conserving surgery. Breast Cancer 2014;21:40-6.

20. START Trialists' Group, Bentzen SM, Agrawal RK, et al. The UK Standardisation of Breast Radiotherapy (START) Trial B of radiotherapy hypofractionation for treatment of early breast cancer: a randomised trial. Lancet 2008;371:1098-107.

21. START Trialists' Group, Bentzen SM, Agrawal RK, et al. The UK Standardisation of Breast Radiotherapy (START) Trial A of radiotherapy hypofractionation for treatment of early breast cancer: a randomised trial. Lancet Oncol 2008;9:331-41.

22. Hannan R, Thompson RF, Chen Y, et al. Hypofractionated 
whole-breast radiation therapy: does breast size matter? Int J Radiat Oncol Biol Phys 2012;84:894-901.

23. Dorn PL, Corbin KS, Al-Hallaq H, et al. Feasibility and acute toxicity of hypofractionated radiation in large-breasted patients. Int J Radiat Oncol Biol Phys 2012;83:79-83.

24. Farace P, Zucca S, Solla I, et al. Planning hybrid intensity

doi: $10.21037 /$ tro.2018.11.01

Cite this article as: Ma L, Hwang A. Improving regional dose distribution while maintaining high delivery efficiency for hypofractionated whole breast radiotherapy. Ther Radiol Oncol 2018;2:60. modulated radiation therapy for whole-breast irradiation. Int J Radiat Oncol Biol Phys 2012;84:e115-22.

25. Mayo CS, Urie MM, Fitzgerald TJ. Hybrid IMRT plans-concurrently treating conventional and IMRT beams for improved breast irradiation and reduced planning time. Int J Radiat Oncol Biol Phys 2005;61:922-32. 